\title{
Adjustable potential probes for band-gap predictions of extended systems through nonempirical hybrid functionals
}

\author{
Thomas Bischoff, Igor Reshetnyak, and Alfredo Pasquarello \\ Chaire de Simulation à l'Echelle Atomique (CSEA), Ecole Polytechnique Fédérale de Lausanne (EPFL), CH-1015 Lausanne, Switzerland
}

(Received 13 February 2019; revised manuscript received 3 May 2019; published 31 May 2019)

\begin{abstract}
We describe a nonempirical procedure for achieving accurate band gaps of extended systems through the insertion of suitably defined potential probes. By enforcing Koopmans' condition on the resulting localized electronic states, we determine the optimal fraction of Fock exchange to be used in the adopted hybrid functional. As potential probes, we consider point defects, the hydrogen interstitial, and various adjustable potentials that allow us to vary the energy level of the localized state in the band gap. By monitoring the delocalized screening charge, we achieve a measure of the degree of hybridization with the band states, which can be used to improve the band-gap estimate. Application of this methodology to AlP, $\mathrm{C}$, and $\mathrm{MgO}$ yields band gaps differing by less than $0.2 \mathrm{eV}$ from experiment.
\end{abstract}

DOI: 10.1103/PhysRevB.99.201114

Density functional theory (DFT) in the semilocal approximation has grown into a standard method for electronic-structure calculations [1,2]. Nevertheless, semilocal functionals suffer from important deficiencies. In particular, the prediction of band gaps remains unsatisfactory [3]. One of the main reasons for this deficiency is the spurious self-interaction of the electron [4], which can more generally be related to the many-body self-interaction error $[5,6]$. This concept is based on the fact that the total energy of the exact exchange-correlation functional is a linear function of the number of electrons between integer values [5]. Combining this condition and Janak's theorem [7], one can conclude that a single-particle level should not change upon its occupation. This property is also known as generalized Koopmans' theorem [5].

In practice, it turned out that most of the commonly used electronic-structure methods do not fulfill Koopmans' condition [8]. In order to overcome this deficiency, considerable efforts have been devoted to enforce the piecewise linearity in approximate density functionals [8-21]. In particular, this has led to an improved description of band gaps, ionization potentials, and excited-state spectra of finite systems [8-10]. The application of the same concept to extended systems has proved more challenging because the developed schemes generally rely on localized states, whereas the band edges of solids are usually delocalized. To incorporate this aspect, various schemes have been proposed, for instance, through maximally localized Wannier functions [10-13], on-site potentials [14] or the analysis of defect states in the frameworks of DFT $+U[15,16]$ and hybrid functionals [16-21].

Recently, several nonempirical procedures to fix the undetermined parameters in hybrid functionals have been introduced, either through Koopmans' condition [16-21] or by accounting for the dielectric response of the material [22-29].

*thomas.bischoff@epfl.ch
While different in their implementation, these two approaches show a close relationship for alkali-halide compounds [17], but a more general connection remains to be demonstrated. In particular, Koopmans' condition applied to defect states and interstitial hydrogen probes has been successful in accurately describing band gaps $[17,19,21]$ and polaronic states $[17,18,20,30,31]$. However, open questions concerning the use of defects within this procedure still remain, such as the dependence of the estimated band gap on the selected defect, the relation to the defect energy level in the band gap, and the implications thereof for the identification of the optimal defect.

In this Rapid Communication, we introduce adjustable potential probes for determining band gaps of extended systems in a nonempirical fashion through the use of Koopmans' condition and a hybrid functional formulation. This allows us to study the band-gap prediction as the defect level varies within the band gap. In particular, we examine the relation between the estimated band gaps and the hybridization with the band-edge states, which we describe through the delocalized screening charge in the simulation cell. We apply the proposed scheme to aluminum phosphide (AlP), diamond (C), and magnesium oxide $(\mathrm{MgO})$, which cover a large range of band gaps and show both covalent and ionic bonding character. The accuracy of the band-gap estimate corresponding to the lowest degree of hybridization is assessed and compared to that achieved with point defects or with the interstitial hydrogen probe.

To determine a hybrid functional that satisfies Koopmans' condition for a specific material, we use the construction scheme illustrated in Fig. 1(a) [17]. We consider the PBE0 $(\alpha)$ family of functionals, which contains a single parameter $\alpha$ representing the fraction of incorporated Fock exchange [32] and reverts to the semilocal Perdew-Burke-Ernzerhof (PBE) functional [2] for $\alpha=0$. Then, upon the insertion of a potential probe in the material, we study the single-particle energy level of the induced localized state as a function of $\alpha$. We carry out calculations in two charge configurations, in which the 


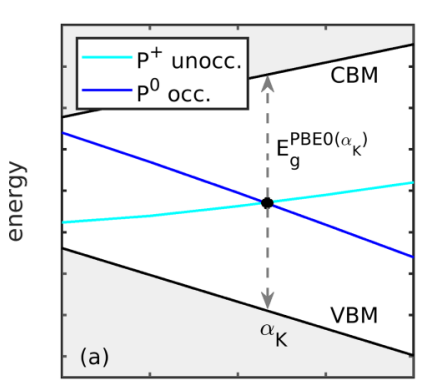

mixing parameter $\alpha$

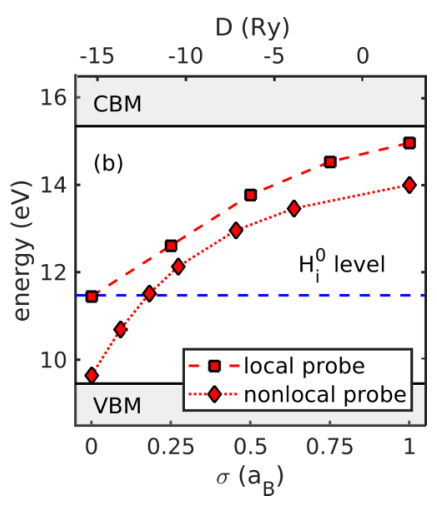

FIG. 1. (a) Occupied and unoccupied single-particle energy levels of a potential probe $\mathrm{P}$ as a function of the mixing parameter $\alpha$ used in the $\operatorname{PBE} 0(\alpha)$ functional. The point of intersection corresponds to the enforcing of Koopmans' condition. The band edges vs $\alpha$ are also shown. The dashed vertical line indicates the resulting band-gap estimate. (b) Defect energy eigenvalue of the local and the lonlocal potential probe in $\mathrm{C}$ as a function of the internal parameters $\sigma$ and $D$, respectively. The energy eigenvalue of the neutral hydrogen interstitial $\mathrm{H}_{\mathrm{i}}{ }^{0}$ is also indicated. The defect eigenvalues and the band-edge levels are obtained with the functional $\operatorname{PBE0}\left(\alpha_{\text {opt }}\right)$, which reproduces the experimental reference for the band gap (see text).

localized state is either occupied or unoccupied. These levels generally evolve linearly with the mixing parameter $\alpha$ and their point of intersection indicates that Koopmans' condition is fulfilled. The corresponding mixing parameter is denoted $\alpha_{\mathrm{K}}$ and is used to obtain an estimate of the band gap with the $\operatorname{PBE} 0(\alpha)$ functional, in which the mixing parameter $\alpha$ is set to $\alpha_{\mathrm{K}}$.

Rather than relying on immutable physical defects, we aim at controlling the energy level of a localized electron state in the band gap. For this purpose, we consider parameterdependent potentials that correspond to generalizations of the standard Coulomb potential of the hydrogen atom. These flexible potential probes are achieved by suitably modifying local or nonlocal pseudopotentials of hydrogen. As a first type of potential probe, we take the local potential

$$
V_{\mathrm{loc}}(r)=-\frac{1}{r} \operatorname{erf}\left(\frac{r}{\sqrt{2} \sigma}\right),
$$

which corresponds to the solution of the Poisson equation for a Gaussian charge distribution of width $\sigma$. For a vanishing $\sigma$, the local potential coincides with the standard Coulomb potential $V_{\mathrm{C}}(r)=-1 / r$. The use of a finite width leads to the transformation of the central divergence into a finite global minimum. The asymptotic $r^{-1}$ behavior is ensured irrespective of the value of $\sigma$. For increasing $\sigma$, the energy level associated with the local potential $V_{\text {loc }}$ shifts upwards and is accompanied by an electronic state of larger extent. The parameter dependence is found to be almost linear when the defect level lies close to the middle of the band gap, as shown in Fig. 1(b). Upon varying $\sigma$, it is possible to position the energy level between the neutral hydrogen level and the conduction-band edge of the host material.

The second type of parametrized potentials that we take under consideration in this work are nonlocal. We define such
TABLE I. Experimental lattice constants $a_{\text {lat }}^{\text {Ext. }}$ used in this work together with calculated and experimental values for the highfrequency dielectric constant $\varepsilon_{\infty}$. Experimental data are taken from Ref. [33].

\begin{tabular}{lcccc}
\hline \hline & & \multicolumn{3}{c}{$\varepsilon_{\infty}$} \\
\cline { 3 - 5 } & $a_{\text {lat }}^{\text {Expt. }}(\AA)$ & Expt. & PBE & PBE0 $(0.25)$ \\
\hline $\mathrm{AlP}$ & 5.464 & 7.50 & 7.87 & 6.93 \\
$\mathrm{C}$ & 3.567 & 5.71 & 5.62 & 5.39 \\
$\mathrm{MgO}$ & 4.216 & 2.94 & 3.05 & 2.81 \\
\hline \hline
\end{tabular}

potentials as

$$
V_{\text {nonloc }}=\tilde{V}_{\text {loc }}(r)+D|\beta\rangle\langle\beta|,
$$

where $\tilde{V}_{\text {loc }}(r)$ is a local Coloumb-like potential, $D$ is a controllable parameter, and $|\beta\rangle$ is a projector on a localized orbital of $s$ symmetry. For a specific value of $D(D=-12.5 \mathrm{Ry})$, the potential probe $V_{\text {nonloc }}$ corresponds to a viable pseudopotential for hydrogen and the eigenvalue describes that of the hydrogen interstitial, as can be seen in Fig. 1(b). The parameter $D$ is then varied to continuously displace the energy level of the nonlocal potential probe either to higher or lower energies compared to the neutral hydrogen level. The energy level is found to shift monotonically with $D$. The use of these two types of adjustable potential probes allow us to generalize the hydrogen probe concept providing an instrument to tune the associated defect level to a desired energy.

In this Rapid Communication, we consider three materials, namely AlP, C, and $\mathrm{MgO}$. We use experimental lattice constants as given in Table I throughout this study. All calculations are performed with the QUANTUM ESPRESSO code [34]. Norm-conserving pseudopotentials are used to describe corevalence interactions $[35,36]$. The point defects are modeled within a 64-atom supercell together with a $2 \times 2 \times 2$ Brillouin zone sampling. We use energy cutoffs of 50, 80, and $100 \mathrm{Ry}$ for $\mathrm{AlP}, \mathrm{C}$, and $\mathrm{MgO}$, respectively, by which the total-energy convergence is assured within $1 \mathrm{meV} /$ atom. Upon the creation of the defect, the electronic structure is calculated fully self-consistently with no structural relaxations allowed. This implies that the long-range screening is entirely described by the high-frequency dielectric constant $\varepsilon_{\infty}$. We account for the spurious interactions with image charges arising from the periodic boundary conditions through state-of-the-art finitesize corrections to the single-particle energy levels [37]. To assure the overall consistency, the corrections are systematically performed with a dielectric constant corresponding to the mixing parameter $\alpha$ used in the electronic-structure calculation. Therefore, we separately calculate $\varepsilon_{\infty}$ with the $\operatorname{PBE} 0(\alpha)$ functional through applying a sawtooth potential to a $1 \times 1 \times 8$ supercell $[38,39]$. The values of $\varepsilon_{\infty}$ calculated with the PBE and PBE0(0.25) functionals are given in Table I. Generally, the dependence of the calculated dielectric constant on the hybrid functional is weak and a good agreement with experimental counterparts is achieved. We verify that the adopted parameters give defect charge distributions localized at the defect site.

It is important to set the references for the band gaps against which we benchmark our calculations. For AlP, C, 

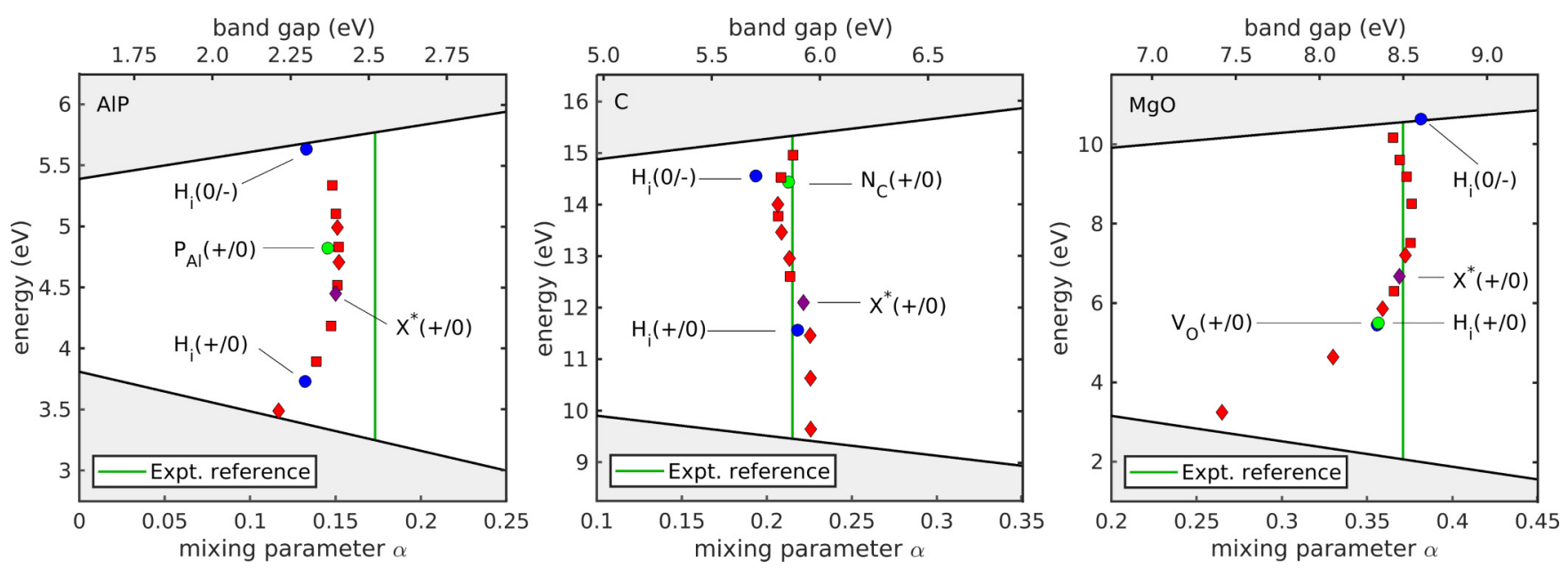

FIG. 2. Band edges and defect energies at the points of intersection displayed vs mixing parameter $\alpha$, as obtained with the PBE0( $\alpha)$ functional for various potential probes in $\mathrm{AlP}, \mathrm{C}$, and $\mathrm{MgO}$. The results for the parametrized local and nonlocal potentials are indicated by red squares and red diamonds, respectively. Additionally, labeled defects comprise $\mathrm{P}_{\mathrm{Al}}$ in $\mathrm{AlP}, \mathrm{N}_{\mathrm{C}}$ in $\mathrm{C}$, and $\mathrm{V}_{\mathrm{O}}$ in $\mathrm{MgO}$, together with two different charge transition levels for the hydrogen interstitial $\mathrm{H}_{\mathrm{i}} . \mathrm{X}^{*}$ refers to the potential probe with the smallest degree of hybridization $\delta$. The indicated band gaps correspond to those obtained with the $\operatorname{PBE} 0(\alpha)$ functional. The vertical green lines correspond to experimental references (see text).

and $\mathrm{MgO}$, we take experimental band gaps $E_{\mathrm{g}}^{\text {Expt. }}$ of 2.50 [40], 5.48 [40], and $7.97 \mathrm{eV}$ [33], respectively. These values correspond to fundamental electronic gaps. To ensure a proper comparison between theory and experiment, we add to the experimental band gaps renormalizations due to phonons, i.e., $0.02 \mathrm{eV}$ for AlP, $0.37 \mathrm{eV}$ for $\mathrm{C}$, and $0.53 \mathrm{eV}$ for $\mathrm{MgO}$, where the former two estimates are based on experimental data [40] and the latter corresponds to a theoretical value [41] and includes the effect of lattice polarization [42]. Further effects such as spin-orbit coupling are neglected. We estimate that the experimental references obtained in this way are subject to a global uncertainty of $0.1 \mathrm{eV}$.

For various point defects, the hydrogen interstitial, and the two types of adjustable potential probes, we determine the points of intersections following the procedure illustrated in Fig. 1. As defect levels, we consider the $(+/ 0)$ level for the $\mathrm{P}_{\mathrm{Al}}$ antisite in AlP, the substitutional $\mathrm{N}_{\mathrm{C}}$ in $\mathrm{C}$, and the oxygen vacancy $\mathrm{V}_{\mathrm{O}}$ in $\mathrm{MgO}$. For the hydrogen interstitial, which is inserted at the center of the largest void, we take under consideration both the $(+/ 0)$ and $(0 /-)$ charge transitions. As far as both the local and nonlocal potential probes are concerned, we position their centers at the same location as that of the hydrogen interstitial. We focus on the $(+/ 0)$ transition and use parameters for which the defect levels sample the band gap. The results are given in Fig. 2. The mixing parameters $\alpha_{\mathrm{K}}$ satisfying Koopmans' condition for the various defects and probes fall in a narrow range for each of the three considered materials, supporting the robustness of the proposed scheme. In particular, the derived $\alpha_{\mathrm{K}}$ values achieved with the two types of adjustable potential probes almost coincide when the associated energy level is the same, and this holds to some extent also for the other defects. More generally, the adjustable potential probes allow us to visualize trends resulting from the continuous variation of the defect energy level in the band gap. We detect a systematic tendency of deviating from the ideal vertical line for defect energies close to the band edges. This deviation is especially evident in the vicinity of the valence band of $\mathrm{MgO}$ [cf. Fig. 2]. For each value of $\alpha$, Fig. 2 also provides the band gap achieved with the $\operatorname{PBEO}(\alpha)$ functional. We note that the band gaps corresponding to $\alpha_{\mathrm{K}}$ values resulting from defect levels close to the middle of the band gap fall within about $0.2 \mathrm{eV}$ from the experimental reference (cf. Fig. 2), consistent with previous observations [17]. However, the behavior close to the band edges leads to a deterioration of this agreement.

It is natural to assign the deviations observed for defect levels falling in the vicinity of the band edges to the hybridization of the defect state with the delocalized band-edge states. To verify this connection, we adopt a criterion for describing the degree of hybridization on the basis of the delocalized screening charge, as proposed in Ref. [43]. Indeed, the polarization charge that screens a charged defect is drawn in a uniform way from the periodically repeated simulation cell $[15,43]$. Far from the defect, this gives an average charge density of $(1-1 / \varepsilon) q / \Omega$, where $\varepsilon$ is the dielectric constant, $q$ the total defect charge, and $\Omega$ the volume of the supercell. In the presence of hybridization, the observed charge density is found to deviate from this reference value [43]. We define the degree of hybridization $\delta$ through this deviation, as shown in Fig. 3(a). We use a Gaussian broadening with a width of $1 \AA$ to smoothen the defect charge density prior to the evaluation of $\delta$.

To demonstrate its direct connection with the deviations in Fig. 2, we evaluate the degree of hybridization $\delta$ for all considered defects versus the corresponding defect energy level within the band gap. The calculated values in the case of $\mathrm{MgO}$ are shown in Fig. 3(b). We observe that the lowest degree of hybridization is found for defect states in the middle of the band gap. The hybridization increases noticeably when the defect level is located in the vicinity of either the valence or the conduction band. Considering both local and nonlocal potential probes, we identify the highlighted defect $\mathrm{X}^{*}$ yielding the minimal degree of hybridization. For the three considered materials, the energy level of $X^{*}$ is found 


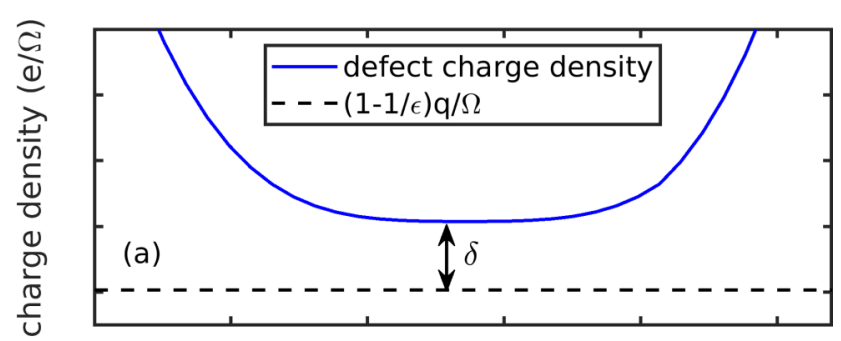

bulk-like supercell region z $(\AA)$

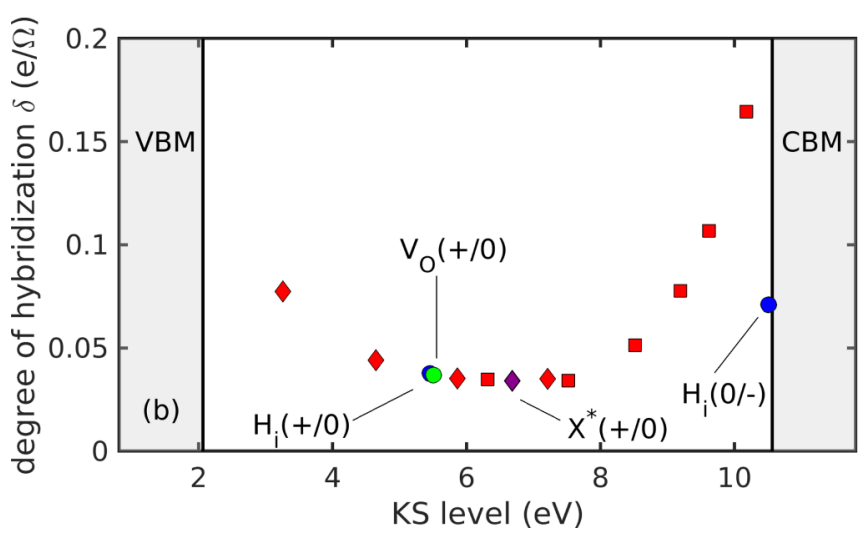

FIG. 3. (a) Schematic view of the degree of hybridization $\delta$ defined in this work as the deviation of the smoothened defect charge density from the delocalized screening charge density (horizontal dashed line) at a large distance from the defect site. (b) Degree of hybridization for various potential probes in $\mathrm{MgO}$ as a function of the defect energy level. The results for the parametrized local and nonlocal potentials are indicated by red squares and red diamonds, respectively. The indicated band-edge energies correspond to the functional $\operatorname{PBE} 0\left(\alpha_{\text {opt }}\right)$, which reproduces the experimental reference for the band gap (see text).

to lie close to the middle of the band gap (cf. Fig. 2). The corresponding mixing parameter $\alpha_{\mathrm{K}}$ then gives an accurate description of the band gap. Given the smooth dependence of the defect level on the internal parameters of the potential probes (cf. Fig. 1), the present scheme is suitable to be used in an automated minimization process. We note that the hybridization between band-edge and defect states depends critically on the host material. In particular, we observe in $\mathrm{MgO}$ a strong interaction with the valence band, which shows a high density of states due to the localized nature of the $\mathrm{O}$ $2 p$ states [44]. Such a dramatic behavior is seen neither for AlP nor for diamond. These results allow us to understand the dependence of the mixing parameter $\alpha_{\mathrm{K}}$ on the considered defect and to improve our selection criteria for identifying the most suitable defect states.

In Table II, we give the band gaps of AlP, $\mathrm{C}$, and $\mathrm{MgO}$ obtained from the $(+/ 0)$ defect level of the potential probe $\mathrm{X}^{*}$ showing the minimal degree of hybridization, in comparison with those obtained from the $(+/ 0)$ and $(0 /-)$ charge transitions of the hydrogen interstitial $\mathrm{H}_{\mathrm{i}}$. All hybrid functionals that
TABLE II. Band gaps (in eV) of AlP, C, and $\mathrm{MgO}$ obtained with the $\operatorname{PBE} 0\left(\alpha_{\mathrm{K}}\right)$ functional, in which the mixing parameter is set through enforcing Koopmans' condition for the two different charge transition levels of the hydrogen interstitial, $\mathrm{H}_{\mathrm{i}}(+/ 0)$ and $\mathrm{H}_{\mathrm{i}}(0 /-)$, as well as for the potential probe $\mathrm{X}^{*}(+/ 0)$ showing the minimal degree of hybridization. The corresponding mixing parameters $\alpha_{\mathrm{K}}$ are given in parentheses. The mean absolute errors (MAEs) are determined with respect to the experimental references (Expt. in last column). These correspond to fundamental band gaps corrected for phonon renormalization.

\begin{tabular}{lcccc}
\hline \hline & $\mathrm{H}_{\mathrm{i}}(+/ 0)$ & $\mathrm{H}_{\mathrm{i}}(0 /-)$ & $\mathrm{X}^{*}(+/ 0)$ & Expt. \\
\hline $\mathrm{AlP}$ & $2.30(0.13)$ & $2.30(0.13)$ & $2.40(0.15)$ & 2.52 \\
$\mathrm{C}$ & $5.90(0.21)$ & $5.71(0.19)$ & $5.93(0.22)$ & 5.85 \\
$\mathrm{MgO}$ & $8.35(0.36)$ & $8.61(0.38)$ & $8.48(0.37)$ & 8.50 \\
$\mathrm{MAE}$ & 0.14 & 0.16 & 0.07 & \\
\hline \hline
\end{tabular}

result from these nonempirical constructions give band gaps in good agreement with experimental references (Table II). The mean absolute errors are within $0.20 \mathrm{eV}$, which is comparable to other state-of-the-art methods for band-gap evaluation [27-29,45,46]. Noticeably, the MAE of the potential probe $\mathrm{X}^{*}$ is lower than those for $\mathrm{H}_{\mathrm{i}}$, indicating that minimizing the degree of hybridization generally improves the bandgap estimate. This property is particularly valuable when the natural defect levels associated with $\mathrm{H}_{\mathrm{i}}$ lie close to the band edges or even outside the band gap. More generally, these results validate a rational procedure for identifying a suitable localized state for band-gap evaluation through the fulfillment of Koopmans' condition. In view of the results in Ref. [21], we expect that such a procedure should also be applicable to systems of lower dimensions.

In this Rapid Communication, we present two types of adjustable potential probes for band-gap evaluations through application of Koopmans' condition. Through the use of these potential probes, the hybridization with the band-edge states can be minimized to yield band gaps within $0.2 \mathrm{eV}$ or lower from experimental values. The dependence on the parameters of the potential probes is smooth and thus suitable to be implemented in an automated minimization process. The present results provide guidelines for the selection of suitable potential probes even when natural defect states cannot be used, thereby enlarging the application scope of the methodology based on the enforcement of Koopmans' condition. This is an important step towards establishing the present technique as a serious alternative with respect to many-body perturbation theory schemes and dielectric-dependent hybrid functionals for robust and accurate band-gap predictions.

This work is supported by the Swiss National Science Foundation (SNSF) under Grant No. 200020-172524. The calculations have been performed at CSCS and at SCITASEPFL.
[1] W. Kohn and L. J. Sham, Phys. Rev. 140, A1133 (1965).
[2] J. P. Perdew, K. Burke, and M. Ernzerhof, Phys. Rev. Lett. 77, 3865 (1996). 
[3] L. J. Sham and M. Schlüter, Phys. Rev. Lett. 51, 1888 (1983).

[4] J. P. Perdew and A. Zunger, Phys. Rev. B 23, 5048 (1981).

[5] J. P. Perdew, R. G. Parr, M. Levy, and J. L. Balduz, Phys. Rev. Lett. 49, 1691 (1982).

[6] P. Mori-Sanchez, A. J. Cohen, and W. Yang, J. Chem. Phys. 125, 201102 (2006).

[7] J. F. Janak, Phys. Rev. B 18, 7165 (1978).

[8] V. Atalla, I. Y. Zhang, O. T. Hofmann, X. Ren, P. Rinke, and M. Scheffler, Phys. Rev. B 94, 035140 (2016).

[9] S. Refaely-Abramson, S. Sharifzadeh, N. Govind, J. Autschbach, J. B. Neaton, R. Baer, and L. Kronik, Phys. Rev. Lett. 109, 226405 (2012).

[10] I. Dabo, A. Ferretti, N. Poilvert, Y. Li, N. Marzari, and M. Cococcioni, Phys. Rev. B 82, 115121 (2010).

[11] N. L. Nguyen, N. Colonna, A. Ferretti, and N. Marzari, Phys. Rev. X 8, 021051 (2018).

[12] J. Ma and L.-W. Wang, Sci. Rep. 6, 24924 (2016).

[13] M. Weng, S. Li, J. Zheng, F. Pan, and L.-W. Wang, J. Phys. Chem. Lett. 9, 281 (2018).

[14] M. Cococcioni and S. de Gironcoli, Phys. Rev. B 71, 035105 (2005).

[15] S. Lany and A. Zunger, Phys. Rev. B 80, 085202 (2009).

[16] S. Lany and A. Zunger, Phys. Rev. B 81, 205209 (2010).

[17] G. Miceli, W. Chen, I. Reshetnyak, and A. Pasquarello, Phys. Rev. B 97, 121112(R) (2018).

[18] S. Kokott, S. V. Levchenko, P. Rinke, and M. Scheffler, New J. Phys. 20, 033023 (2018).

[19] P. Deák, Q. Duy Ho, F. Seemann, B. Aradi, M. Lorke, and T. Frauenheim, Phys. Rev. B 95, 075208 (2017).

[20] B. Sadigh, P. Erhart, and D. Åberg, Phys. Rev. B 92, 075202 (2015).

[21] T. J. Smart, F. Wu, M. Govoni, and Y. Ping, Phys. Rev. Mater. 2, 124002 (2018).

[22] T. Shimazaki and Y. Asai, Chem. Phys. Lett. 466, 91 (2008).

[23] T. Shimazaki and Y. Asai, J. Chem. Phys. 130, 164702 (2009).

[24] T. Shimazaki and Y. Asai, J. Chem. Phys. 132, 224105 (2010).

[25] A. Alkauskas, P. Broqvist, and A. Pasquarello, Phys. Status Solidi B 248, 775 (2011).
[26] M. A. L. Marques, J. Vidal, M. J. T. Oliveira, L. Reining, and S. Botti, Phys. Rev. B 83, 035119 (2011).

[27] J. H. Skone, M. Govoni, and G. Galli, Phys. Rev. B 89, 195112 (2014).

[28] J. H. Skone, M. Govoni, and G. Galli, Phys. Rev. B 93, 235106 (2016).

[29] W. Chen, G. Miceli, G.-M. Rignanese, and A. Pasquarello, Phys. Rev. Mater. 2, 073803 (2018).

[30] F. Ambrosio, J. Wiktor, F. De Angelis, and A. Pasquarello, Energy Environ. Sci. 11, 101 (2018).

[31] A. R. Elmaslmane, M. B. Watkins, and K. P. McKenna, J. Chem. Theory Comput. 14, 3740 (2018).

[32] J. P. Perdew, M. Ernzerhof, and K. Burke, J. Chem. Phys. 105, 9982 (1996).

[33] O. Madelung, Semiconductors: Data Handbook (Springer, Berlin, 1996).

[34] P. Giannozzi, S. Baroni, N. Bonini, M. Calandra, R. Car, C. Cavazzoni, D. Ceresoli, G. L. Chiarotti, M. Cococcioni, I. Dabo et al., J. Phys.: Condens. Matter 21, 395502 (2009).

[35] M. van Setten, M. Giantomassi, E. Bousquet, M. Verstraete, D. Hamann, X. Gonze, and G.-M. Rignanese, Comput. Phys. Commun. 226, 39 (2018).

[36] D. R. Hamann, Phys. Rev. B 88, 085117 (2013).

[37] W. Chen and A. Pasquarello, Phys. Rev. B 88, 115104 (2013).

[38] K. Kunc and R. Resta, Phys. Rev. Lett. 51, 686 (1983).

[39] R. Resta and K. Kunc, Phys. Rev. B 34, 7146 (1986).

[40] M. Cardona and M. L. W. Thewalt, Rev. Mod. Phys. 77, 1173 (2005).

[41] J. P. Nery, P. B. Allen, G. Antonius, L. Reining, A. Miglio, and X. Gonze, Phys. Rev. B 97, 115145 (2018).

[42] W. R. L. Lambrecht, C. Bhandari, and M. van Schilfgaarde, Phys. Rev. Mater. 1, 043802 (2017).

[43] H.-P. Komsa, T. T. Rantala, and A. Pasquarello, Phys. Rev. B 86, 045112 (2012).

[44] R.-Q. Zhang, C.-E. Kim, B. Delley, C. Stampfl, and A. Soon, Phys. Chem. Chem. Phys. 14, 2462 (2012).

[45] M. Shishkin, M. Marsman, and G. Kresse, Phys. Rev. Lett. 99, 246403 (2007).

[46] W. Chen and A. Pasquarello, Phys. Rev. B 92, 041115(R) (2015). 